\title{
An Evaluation and Comparison Study for Online Tourism E-commerce Websites between East and West
}

\author{
Tianxiang Zheng \\ Shenzhen Tourism College of Jinan University, Shenzhen, Guangdong, 518053, China \\ zheng_tx@jnu.edu.cn
}

\begin{abstract}
Academic researchers have long advocated the importance of assessing the tourism websites. On the basis of predecessors' work, this study proposes an evaluation system of tourism e-commerce websites by the methodologies of AHP(Analytic Hierarchy Process) and TOPSIS(Technique for Order Preference by Similarity to an Ideal Solution). Five famous online websites, including expedia.com, orbitz.com, travelocity.com, ctrip.com and tuniu.com, were selected as examples for examination and comparison. The results show that expedia.com and ctrip.com are the leading websites for abroad and domestic China respectively, which conforms to the real world phenomenon. Suggestions for improvement derived from the results are also included. Not only can this study help owners of practitioners to assess his/her own website in an objective manner, but also recognize the gap between the top leaders of the industry so as, ultimately, to improve the website quality and operational practice.
\end{abstract}

Keywords: Website Evaluation, Tourism E-commerce Website, AHP, TOPSIS

\section{国内外在线旅游电子商务网站的评价与比较}

\author{
郑天翔 \\ 暨南大学深圳旅游学院, 南山区, 深圳, 广东, 中国 \\ zheng_tx@jnu.edu.cn
}

中文摘要. 旅游网站评价一直是国内外学 术界关注的问题。本文在整理前人相关研究 的基础上, 将层次分析法AHP (Analytic Hierarchy Process) 和理想解近似度顺序偏 好法TOPSIS (Technique for Order Preference by Similarity to an Ideal Solution）引入旅游 网站评价中, 并选取国内外五个知名的在线 旅游电子商务网站进行数值求解和比较分 析，包括 expedia.com、 orbitz.com、 travelocity.com、ctrip.com (即携程网) 和 tuniu.com（即途牛网）。结果表明, expedia.com和携程网分别是国内外同类网 站中的佼佼者, 这与现实情形非常吻合。同 时, 论文根据求解结果对这几个网站的改进 分别给出了相应的对策和建议。本文的研究 成果能帮助网站运营者或从业者客观地评 价自身网站的优劣, 并获知与行业领先者的 差距, 从而有针对性地提高网站质量, 改善 运作业务。

关键词：网站评价; 旅游电子商务网站; AHP; TOPSIS 


\section{1. 引言}

旅游电子商务网站作为旅游信息化最 广泛、最直接和最有效的手段, 对旅游业的 发展起着至关重要的作用, 是国内外旅游业 进行资源整合、管理和营销的重要工具。据 统计, 目前中国在线旅游市场交易非常活跃 [1], 越来越多的消费者直接通过互联网预订 旅游服务产品。网站作为旅游企业开展电子 商务的重要平台, 如何有效地向用户呈现有 特色、有价值的旅游信息, 并促成最终交易, 是每个旅游电子商务网站建设和运营必须 考虑的首要问题和努力方 向 $^{[2]}$ 。

自从 $\mathrm{Lu}^{[3]}$ 首次提出网站评价方法以来, 国内外很多学者对旅游相关网站的评价方 法做了探讨。其中, 国外学者研究对象涉及

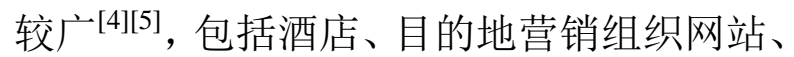
旅游公司和在线旅游网站等, 研究角度也较

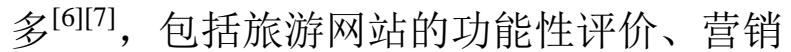
效果评价和使用者满意度、行为或动机三个 方面。这些方法各有特点, 从不同侧面推动 了旅游网站评价领域的发展, 并丰富了学者 和从业者对旅游网站的认识。经过十几年的 发展, 国外对旅游网站的研究体系正逐渐形 成, 研究切入点多样化, 研究方法也较为丰 富。相比之下, 国内关于旅游网站评价的研 究不太多, 仅有零散的几个重要工作 [8][9][10][11][12][13], 在研究方法和切入点上还存 在一定差距, 主要以描述性定性研究为主, 定量研究和理论研究较少。本文选取在网上 旅游市场上占主导地位 ${ }^{[9]}$ 的在线旅游电子 商务网站为研究对象, 站在运营者的角度, 试图运用运筹学方法构建一个较实用的评 价模型, 将使用者感知与网站属性结合起来 帮助运营者识别自身网站存在的优劣势, 并 通过客观比较得出可操作的有指导性的结 论。

\section{2. 研究方法}

本文构建的评价模型其基本思想是首 先界定评价的指标, 然后再进行决策比较。 为此, 首先选取层次分析法 (Analytic Hierarchy Process, 简称AHP) ${ }^{[14]}$ 建立旅游 网站指标评价体系, 再借助用于多目标决策 分析的理想解近似度顺序偏好法
( Technique for Order Preference by Similarity to an Ideal Solution, 简称TOPSIS ) [15]来获得旅游网站在这些指标上的评价。

\subsection{AHP指标体系的构建}

结合前人在电子商务网站上的研究成

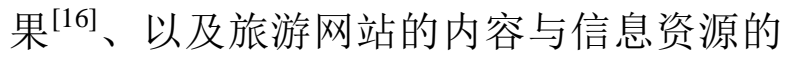
特点等因素, 本文把在线旅游电子商务网站 评价分解为受欢迎程度、服务多样性、服务 便捷性三大评价准则, 并在此基础上对每一 准则分别提取其影响因素（评价因子），同 时把 “网站性能” 和 “服务质量” 这两大关 键要素紧密联系起来, 最终得到一个较为全 面、各指标独立的 “目标层一准则层一因子 层” 指标体系。体系的层次结构如表1所示。

表1 评价指标体系

\begin{tabular}{|c|c|c|}
\hline 目标层 & 准则层 & 因子层 \\
\hline \multirow{10}{*}{$\begin{array}{c}\text { 在线旅游电子商务网 } \\
\text { 站评价指标体系 }\end{array}$} & \multirow{3}{*}{ B1.受欢迎度 } & C1. 日均 PV 值 \\
\hline & & C2. 日 IP 值 \\
\hline & & C3.PR 值 \\
\hline & \multirow{3}{*}{ B2. 服务多样性 } & C4.预订服务多样性 \\
\hline & & C5.信息提供多样性 \\
\hline & & C6.营销方式多样性 \\
\hline & \multirow{4}{*}{ B3. 服务质量 } & C7. 访问速度 \\
\hline & & $\begin{array}{c}\text { C8.获取信息的便捷 } \\
\text { 度 }\end{array}$ \\
\hline & & C9.页面设计 \\
\hline & & C10. 安全与隐私 \\
\hline
\end{tabular}

指标体系中各因子内涵解读如下:

受欢迎度(B1): 反映了网站本身的质量 和网站在社会公众心目中的地位;

日均PV值 $(\mathrm{C} 1)$ : 平均每天该网站所访 问的页面数;

日IP值(C2): 平均每天访问网站的独立 IP数, 日IP可以大致表现出访问网站的人 数;

PR值(C3)：PR 全称PageRank, 即网页 级别, 是一种由搜索引擎根据网页之间相互 的超链接计算的网页排名技术, Google用它 来体现网页的相关性和重要性, 数值在 1 到 10之间;

服务多样性(B2): 反映了网站所提供的 服务的内容的丰富度和形式的多样性。多样 性高的网站往往能适应更为广泛的访问者, 迎合他们的个性化的需求;

预订服务多样性(C4): 网站提供的预订 服务的种类，如预订酒店、航班、门票等; 
信息提供多样性(C5): 网站是否提供天 气、交通、地图等旅游者需要的信息;

营销方式多样性(C6): 网站是否使用微 博、虚拟社区、Email营销、手机客户端等 方式进行营销;

服务质量(B3): 衡量网站质量最重要的 因素, 直接关系到用户的数量和黏性, 以及 网站的生存和发展;

访问速度(C7): 网站的访问速度如何, 网站的下载速度与其他网站相比处于什么 地位;

获取信息的便捷程度 $(\mathrm{C} 8)$ : 网站是否采 取有效的信息组织方式, 使得用户在获取信 息时能够尽可能少的遇到障碍;

页面设计 $(C 9)$ ：页面是否大方、美观、 符合用户的阅读习惯;

安全与隐私 $(\mathrm{C} 10)$ ：网站的交易安全是 否得到保障; 是否有隐私和安全策略, 如果 有, 是否全面和完善。

\section{2 权重的确定}

根据AHP方法的原理，首先运用 “1 9 标度方法” , 并通过文献调查和专家反复讨 论, 对准则层和因子层的因素进行两两判 断, 得到若干判断矩阵。然后, 计算出各矩 阵的最大特征根和相应的归一化特征向量, 并进行一致性检验。检验通过以后, 通过各 指标因子层因素的权重与准则层因素的权 重相乘便可以得出旅游网站综合评价指标 总排序结果, 如表2所示。

表2 评价指标体系权重

\begin{tabular}{|c|c|c|c|}
\hline 目标层 & 准则层 & 因子层 & $\begin{array}{l}\text { 合成权 } \\
\text { 重 }\end{array}$ \\
\hline \multirow{10}{*}{$\begin{array}{c}\text { 在线旅游电子商 } \\
\text { 务网站评价指标 } \\
\text { 体系 }\end{array}$} & \multirow{3}{*}{$\begin{array}{c}\text { B1.受欢迎度 } \\
(0.291)\end{array}$} & $\begin{array}{c}\text { C1. 日均 PV 值 } \\
(0.268)\end{array}$ & 0.078 \\
\hline & & $\begin{array}{c}\text { C2. 日 IP 值 } \\
(0.614)\end{array}$ & 0.187 \\
\hline & & C3.PR 值(0.117) & 0.034 \\
\hline & \multirow{3}{*}{$\begin{array}{c}\text { B2.服务多样 } \\
\text { 性(0.103) }\end{array}$} & $\begin{array}{c}\text { C4.预订服务多 } \\
\text { 样性(0.649) }\end{array}$ & 0.067 \\
\hline & & $\begin{array}{c}\text { C5.信息提供多 } \\
\text { 样性(0.279) }\end{array}$ & 0.029 \\
\hline & & $\begin{array}{c}\text { C6.营销方式多 } \\
\text { 样性(0.072) }\end{array}$ & 0.007 \\
\hline & \multirow{4}{*}{$\begin{array}{c}\text { B3. 服务质量 } \\
(0.605)\end{array}$} & $\begin{array}{c}\text { C7. 访问速度 } \\
(0.263)\end{array}$ & 0.159 \\
\hline & & $\begin{array}{l}\text { C8.获取信息的 } \\
\text { 便捷度 }(0.564) \\
\end{array}$ & 0.341 \\
\hline & & $\begin{array}{c}\text { C9.页面设计 } \\
(0.118)\end{array}$ & 0.071 \\
\hline & & $\begin{array}{c}\text { C10. 安全与隐私 } \\
(0.055)\end{array}$ & 0.033 \\
\hline
\end{tabular}

\section{3 样本数据收集}

为了得到这五个网站在上述十个指标 上的数据, 需要进行资料收集和统计, 收集 时间从2014年8月5日至2014年8月27日。这 十项指标中, 部分数据来自专门的网站统计 工具, 另一部分数据则是由咨询专家后制订 的评分规则得出, 还有一些来自专家打分, 并辅之以对普通用户的问卷调查。

\subsection{TOPSIS求解}

根据TOPSIS原理，第一步是将表的各指 标原始值归一化为效用值, 得到的结果参见 表3。第二步是确定求解各指标的正负理想 解。根据表3中各个因素指标的效用值, 显 然各指标的正负理想解分别 1 和 0 , 且都是正 向指标。第三步是计算各因素指标的效用值 与正负理想解的距离。根据表 2 中的各指标 权重值和表3中各网站的指标效用值, 得到 的最终结果如表4所示。

\section{表3 各样本网站在指标体系下的效用值}

\begin{tabular}{|c|c|c|c|c|c|}
\hline 指标 & expedia & orbitz & travelocity & ctrip & tuniu \\
\hline C1 & 1 & 0.217 & 0.326 & 0.8046 & 0 \\
\hline C2 & 1 & 0.251 & 0.306 & 0.388 & 0 \\
\hline C3 & 1 & 0 & 0 & 1 & 0 \\
\hline C4 & 1 & 0.5 & 1 & 1 & 0 \\
\hline C5 & 0.5 & 0.5 & 0.5 & 1 & 0 \\
\hline C6 & 1 & 0.75 & 0 & 0.75 & 1 \\
\hline C7 & 0.019 & 0 & 0.415 & 0.755 & 1 \\
\hline C8 & 1 & 0.667 & 0.333 & 0.667 & 0 \\
\hline C9 & 1 & 0.5 & 0.5 & 0 & 0.5 \\
\hline C10 & 1 & 1 & 1 & 0 & 1 \\
\hline
\end{tabular}

表4 求解结果

\begin{tabular}{|c|c|c|c|c|}
\hline expedia & orbitz & travelocity & ctrip & tuniu \\
\hline 0.694 & 0.432 & 0.424 & 0.587 & 0.349 \\
\hline
\end{tabular}

\section{4. 对策与建议}

从表 4 来看, 本文选取的 5 个样例中, expedia和携程网 (ctrip) 的总体评分最高, 这恰恰与现实情形十分吻合, 这两个网站分 别代表了国外和国内在线旅游电子商务网 站的最高水平。相比之下, orbitz 和 travelocity次之, 途牛网 (tuniu) 排名最后。 
值得注意的是, 表4揭示的只是各网站的 总体评价, 事实上, 可以根据表 3 数据作进 一步分析，这将在下文中叙述。

\section{1 在评价准则层上的纵向比较}

受欢迎程度 (C1-C3) : expedia在五个网 站中最受欢迎。Expedia网站创办于1995年, 具有最为悠久的历史、广泛而众多的用户、 较高的知名度和权威性。从网站的受欢迎程 度来看, 该网站占据着大量的市场份额, 是 众多旅游者出行的首选网站。国内网站中, 携程网凭借国内巨大的市场, 虽然建站时间 不算早, 但依然大受欢迎。相比之下, orbitz 和travelocity受欢迎程度得分较低。以日IP 为例, 二者连expedia的一半都不及。这可 能是网站建站时间较之于 expedia晚, 同时 作为竞争对手的后者过于强大的缘故。类似 的情况在国内的途牛网也遇到。

服务多样性（C4-C6）：国外的expedia 和国内的携程网提供的服务较为丰富, 拥有 包括机票、宾馆、租车、游轮和门票预订在 内的多种预订功能, 能够向用户提供目的地 的多方面信息, 并通过微博、Email、社交 网络、电话、手机app、自建社区、网上杂 志等方式与用户进行互动, 能比较有效地迎 合不同旅游者的个性化需求。orbitz也拥有 不错的表现, 相比之下, 其余两个网站在这 方面有待提高。

服务质量（C7-C10）：国外的expedia的 服务质量最高, 除访问速度较慢外, 其余指 标均代表行业的最高水平。orbitz、 travelocity和途牛次之, 携程最差。携程的 不足主要集中在页面设计和安全隐私上, 这 将在下一节详细说明。

\section{2 在研究对象上的横向分析与建议}

expedia: 在 10 个选取的指标中, 有 8 个高 居榜首, 在信息提供多样性上也只比携程网 稍差一点 (没有提供天气信息), 稍嫌不足 的是访问速度。作为全球领先的在线旅游公 司, expedia已经在网站各方面做出了顶尖 水平, 至少在本文选取的指标范围上如此。

orbitz: 作为廉价机票、酒店、租车等服 务于一体的网站新贵, 除了日均PV值和日 IP值稍逊于expedia外, orbitz其余指标基本 上与后者相当, 大有后来居上之势。由于在
网站功能、目标用户等方面的同质性, 要与 已经根深蒂固的行业霸主expedia竞争, 必 须突出自己的特色, 才能发现新需求, 探索 新用户。此外, 与expedia一样, 访问速度 也是亟需加强的。

travelocity: 作为拥有最大规模的旅游网 站, 借助其强大的技术优势, travelocity能 提供许多独特的预订服务 (例如火车票预订 服务), 但由于只提供email和电话这两种 营销方式, 因此在营销方式多样性上比较欠 缺。另外, 在获取信息的便捷度上也需多做 功夫。

携程：作为国内的行业老大，携程网在 很多方面都做得非常出色, 但也存在改进的 空间。首先页面设计不理想, 尤其是色彩运 用与网站旅游的主题不太相符, 其次是作为 一个大型网站, 携程网竟然缺乏隐私策略。 虽然国内用户目前对隐私策略并不太重视, 但作为衡量网站安全性的一个指标, 完善的 隐私策略必然会得到逐渐成熟的国内网民 的青睐。此外, 营销方式的多样性也需要与 时俱进, 诸如社交网络、自建社区和有奖活 动等方式应多加考虑。

途牛：除受欢迎程度不太够以外，服务 和信息多样性还有待提高。作为以在线预订 为主要服务项目的旅游电子商务网站, 服务 的多样性直接关系到用户的数量和黏性, 目 前途牛没有提供航班和租车服务, 这是未来 发展需要加强的地方。类似的问题还包括信 息提供多样性, 天气、地图或交通信息可考 虑补充。此外, 途牛网还需要提高自己的服 务质量, 比如, 需要完善信息组织结构, 提 高搜索质量, 以方便用户更轻松地找到所 需。

\section{5. 结束语}

本文对在线旅游电子商务网站的评价 做了研究, 提出了一个基于AHP和TOPSIS 方法的评价模型, 旨在为该领域的发展提供 新的研究思路。针对目前国内旅游电子商务 网站在指标体系的建立和同类网站的比较 上尚无重要工作的现状, 从受欢迎程度、服 务多样性和服务质量入手, 利用AHP方法构 建旅游网站评价的指标体系, 并选择有影响 力的五个在线旅游电子商务网站, 借助 
TOPSIS 方法得到各类网站在上述指标上的 具体评分, 从而获得整体评价, 最终为各网 站提供具有针对性的建议与对策。

\section{致谢}

本文为国家社会科学基金青年项目《流 动性结构失衡背景下的我国金融脆弱性与 金融风险管理研究》（14CJY004）和国家 自然科学基金青年科学基金项目《基于时空 分流导航理论的景区智能化客流引导仿真 系统的原型设计与实证研究》（41201145） 的阶段性成果之一。

\section{References}

[1] C. H. Wang, Y. Zhu. Evaluation and Analysis of Content Delivery Performance of Tourism E-commerce sites. Journal Beijing International Studies University, no.7, pp.1-9, 2014.

[2] C. L. Zhou, J. Yang, Z. Y. Yu Zhao. Study on the Content Delivery Survey and Evaluation of Tourism E-commerce Websites in China. Tourism Tribune, vol. 25, pp. 37-43, 2010.

[3] M. T. Lu, W. Yeung. A framework for effective commercial Web application development. Internet Research, vol.8, pp. 166-173, 1998.

[4] A. M. Morrison, J. S. Taylor, A. Douglas. Website evaluation in Tourism and hospitality. Journal of Travel \& Tourism Marketing, vol.17, pp.233-251, 2004.

[5] D. Buhalis, R. Law. Progress in information technology and tourism management: 20 years on and 10 years after the Internet -The state of eTourism research. Tourism Management, vol. 29, pp.609-623, 2008.

[6] R. Law, S. S. Qi, D. Buhalis. Progress in tourism management: A review of website evaluation in tourism research. Tourism Management, vo.31, pp.297-313, 2010.
[7] C. Ip, R. Law, H. A. Lee. A review of website evaluation studies in the tourism and hospitality fields from 1996 to 2009. International Journal of Tourism Research, vol.13, pp. 234-265, 2011.

[8] J. X. Zhang. Quantitative Evaluation Method on Effectiveness of Tourism Web Sites. Journal of China University of Geosciences (Social Sciences Edition), vol.1, pp. 30-33, 2001.

[9] S. Wu, H. H. Ling. A Study on the Content Delivery Performance of www.ctrip.com, www.China-sss.com, www.elong.com. Tourism Tribune, vol.20, pp.66-69, 2005.

[10]Z. Lu, Y. L. Li. A Supply-demand Difference Study of Tourism Online Service Satisfaction in Beijing. Economic Geography, vol.25, pp.732-735, 2005.

[11]X. C. Wan. A Preliminary Study on the Evaluation of Hotel Websites Based on Tourist Service Function: A Case Study of Star-rated Hotels in Nanjing. Tourism Tribune, vol.22, pp.64-67, 2007.

[12]W. Xiong, L. W. Ye. A study on function evaluation of virtual tour websites in China. Human Geography, vol.26, pp.154-160, 2011.

[13]Y. Y. Lv, K. Bai. A Study of Tourism Trading Website Consumer Evaluation: A Case Study of Ctrip. Tourism Science, vol. 28, pp.49-60, 2014.

[14]T. L. Saaty. Analytic hierarchy process. New York: McGraw-Hill, 1980.

[15]C. L. Hwang, K. Yoon. Multiple attribute decision making: methods and applications: a state-of-the-art survey. New York: Springer-Verlag, 1981.

[16]L. Cai, J. A. Card, S. T. Cole. Content delivery performance of world wide web sites of US tour operators focusing on destinations in China. Tourism Management, vol.25, pp.219-227, 2004. 